\title{
Drugs in cerebral and peripheral arterial disease
}

\author{
Gordon D O Lowe
}

Stroke, intermittent claudication, foot ischaemia, and Raynaud's phenomenon are common and disabling diseases. Compared with other cardiovascular disorders, however, their treatment has been relatively neglected by doctors and clinical pharmacologists, partly because of a lack of facilities. It is paradoxical that young patients with critical strokes are "abandoned" in the corners of general wards, often with little attention to medical treatment, whereas patients 30 years older (and often less ill) are rushed to coronary care units for intensive monitoring and treatments that require such specialised units to show their efficacy. It is equally strange that patients with peripheral arterial disease are routinely referred to surgeons, who operate on only a minority. The United Kingdom and United States of America, traditionally performers of randomised controlled trials, lack stroke units and angiologists who receive unselected referrals. The paucity of large randomised studies is therefore not surprising.

Few "new" drugs have been approved in recent years for treating cerebral or peripheral arterial disease in the United Kingdom, although several are under clinical trial and some may soon receive product licences. Several "old" drugs, however, have recently been evaluated further in randomised controlled trials of reasonable size, which will be reviewed, though few trials are large enough to give definite conclusions about whether treatments are beneficial, harmful, or of no value. Overviews and meta-analyses may help clinicians to make provisional assessments in the meantime.

\section{Potential sites of action ARTERIES}

Most drugs traditionally promoted for cerebral or peripheral arterial disease are vasodilators. There is little theoretical rationale for dilating larger vessels, except in Raynaud's disease and in subarachnoid haemorrhage, in which large vessel spasm has been found. Furthermore, doses that produce systemic vasodilatation may "steal" blood from ischaemic areas and also produce systemic adverse effects (table I). On the other hand, ischaemia of the brain or limbs is not a simple plumbing problem of large vessel diameter (table II). Large vessel occlusion arises not only from spasm and atherosclerotic stenoses but also from thromboemboli of platelets and fibrin. Potential roles therefore exist for platelet inhibitors, anticoagulants, thrombolytic agents, and drugs that enhance endogenous fibrinolysis.

\section{MICROCIRCULATION}

Ischaemia depends ultimately on disturbance of nutritive microvascular flow rather than macrovascular obstruction. Vasoactive drugs may prevent or improve the microcirculatory flow disturbance that succeeds large vessel occlusion-for instance, by direct action on small vessels. Many vasoactive drugs affect not only vessels but also the flow or interaction with vessel walls, or both, of circulating blood cells (table II). This is not surprising considering that vessels and cells share a common mesenchymal origin and several common chemical mediators (such as serotonin, prostacyclin). The intrinsic flow resistance of blood (its rheological behaviour) deteriorates in ischaemia and is predictive of adverse prognosis: several drugs have potentially beneficial rheological effects that may increase microvascular flow. Such effects include increased red cell deformability (in capillaries) and decreased red cell aggregation (in venules) under ischaemic conditions; reduction in platelet microaggregates, which can block arterioles and capillaries; and decreased activation of white cells, which may obstruct capillary or venular flow, adhere to endothelium, and damage cells by producing lysosomal enzymes, leukotrienes, or toxic oxygen derivatives.

\section{TISSUE CELLS}

Finally, drugs may act by protecting brain or limb cells from ischaemic damage, rather than by increasing microvascular flow. Possible mechanisms for such "cytoprotection" include protection against toxic products of ischaemia (for example, by scavengers of toxic oxygen products); reduction of calcium influx (for example, by calcium channel blockers); increased oxidative metabolism with reduction of lactic acidosis (for example, by naftidrofuryl); and decreased oedema (for example, by hyperosmotic solutions of mannitol, sorbitol, or glycerol).

\section{Cerebral arterial disease}

ACUTE ISCHAEMIC (OR UNSELECTED) STROKE ${ }^{12}$

As with myocardial infarction, specific treatment of acute cerebral infarction is most likely to benefit the patient when given in the first few hours, before infarction of the whole ischaemic area: one should not wait an arbitrary 24 hours to let a "transient ischaemic attack" become a "completed stroke." An accurate diagnosis should be made, by computed tomography as well as clinical evaluation, to identify the sizable minority of patients with intracranial haemorrhages, tumours, or lacunar infarctions and make them secondary exclusions from studies of major cerebral infarction. The most relevant end points to the patients are death and long term (several months) or short term (one month) dependence. Unfortunately, most reported studies have been too small, have not included routinely performed computed tomography, and have reported the outcomes as neurological scores, which interest neurologists, rather than activities of daily living, which interest patients. ${ }^{12}$

\section{Isovolaemic haemodilution with dextran $40^{3}$}

Several small studies have suggested that dextran infusions may be beneficial in acute cerebral infarction, possibly by lowering the packed cell volume and thus blood viscosity. Two recently reported large, multicentre, randomised, controlled trials from Italy (1267 patients) and Scandinavia (373 patients) assessed the value of isovolaemic haemodilution (by venesection and dextran 40 infusion) in acute cerebral infarction, which had been confirmed by computed tomography in almost all patients. Mean packed cell volume was reduced from $43-44 \%$ to $37-38 \%$. As no benefit was
Gordon D O Lowe, MD, senior lecturer and consultant 
TABLE I - Adverse effects of vasodilators and cautions in their use

\begin{tabular}{ll}
\hline Adverse effects & Cautions \\
\hline $\begin{array}{l}\text { Hypotensive dizziness and faints, } \\
\text { especially postural and exercise } \\
\text { induced }\end{array}$ & $\begin{array}{l}\text { Angina, recent myocardial } \\
\text { infarction } \\
\text { Tachycardia, palpitations }\end{array}$ \\
$\begin{array}{l}\text { Bradycardia, heart block } \\
\text { Flushing, headache }\end{array}$ & Othent haemorrhage \\
Nausea, vomiting & \\
\hline
\end{tabular}

TABLE II - Potential sites of action for drugs to treat ischaemia

\begin{tabular}{lll}
\hline Sites & Conditions & Drugs \\
\hline Arteries & Atherosclerosis & Lipid reducers \\
& Plood pressure reducers \\
& Platelet or fibrin thrombus & Anticoagulants \\
& & Thrombolytic agents \\
& & Stimulators of endogenous fibrinolysis \\
& Arterial and collateral vessel smooth muscle & Vasodilators \\
Arterioles & Arteriolar smooth muscle & Vasodilators \\
& Platelet aggregates & Antiplatelet agents \\
Capillaries & Platelet aggregates & Antiplatelet agents \\
& White cell plugging & White cell antiactivators \\
& Red cell deformation & Haemorheological agents \\
Venules & Red cell aggregation & Haemorheological agents \\
& White cell adhesion & White cell antiactivators \\
Tissue cells & Calcium influx & Calcium channel blockers \\
& Oxygen radical damage & Free radical scavengers \\
& Metabolic derangement & Metabolic regulators \\
& Oedema & Hyperosmotic solutions \\
\hline
\end{tabular}

observed in either study, we can be confident the routine application of this regimen is not useful for treating acute cerebral infarction. Possible explanations include the facts that more patients with stroke die only if they have high (over $48-50 \%$ ) packed cell volumes; dextran increases plasma viscosity; and cardiac output in elderly patients with stroke fails to increase sufficiently to compensate for the reduction in haemoglobin concentration and thus in oxygen transport. Future studies of haemodilution should evaluate patients with high packed cell volumes; plasma expanders such as hetastarch, which do not increase plasma viscosity; and haemodynamic monitoring. ${ }^{3}$

\section{Glycerol}

Glycerol may be beneficial in acute infarction by reducing cerebral oedema. An analysis of the eight published randomised controlled trials found that treatment reduced the risk of short term (within six weeks) death by about $36 \%$ ( $95 \%$ confidence interval $-4 \%$ to $-58 \%)$. $^{1}$ Analysis of the four trials that reported longer follow up found a non-significant reduction in death rates of $21 \%(95 \%$ confidence interval $-51 \%$ to $+28 \%) .{ }^{1}$ Further studies of larger numbers, diagnosed by computed tomography and followed up for long term disability as well as death, are required.

\section{Naftidrofuryl}

This drug has several actions, including increased oxidative metabolism by ischaemic cells with reduction of lactic acidosis. An analysis of the two published studies of acute stroke ( 100 and 89 patients respectively) found a $29 \%$ reduction in death rates within three months, but with wide confidence intervals. ${ }^{1}$ The second, recent study used computed tomography to establish the diagnosis of infarction. Further larger studies are again required.

\section{Nimodipine}

This calcium channel blocker has a greater antivasoconstrictive effect on cerebral than limb arteries, crosses the blood brain barrier, and may also reduce ischaemic brain cell damage. A controlled trial of
186 patients with acute ischaemic stroke, confirmed by computed tomography, observed a $70 \%$ reduction in death rate within four weeks $(95 \%$ confidence interval $-12 \%$ to $-90 \%$ ), with the death rate difference maintained at six months.' All patients in this study also received dextran and heparin. Larger studies of nimodipine are in progress. Nicardipine is another calcium channel blocker also under evaluation for treating cerebral ischaemia.

\section{$\beta$ Adrenergic blockers}

A study of 302 conscious patients with clinically diagnosed hemisphere strokes (few underwent computed tomography) randomised to receive atenolol (50 mg daily), slow release propranolol ( $80 \mathrm{mg}$ daily), or placebo, all starting within 48 hours, showed no significant differences in death or disability at six month follow up, but again the 95\% confidence intervals were wide. ${ }^{4}$ Larger studies with computed tomography are therefore required.

\section{Corticosteroids}

Corticosteroids may reduce cerebral oedema and were assessed in several small studies between 1956 and 1976. Two more recent randomised trials of over 100 patients found no benefit in death or disability. The first trial did not use computed tomography and had a one year follow up; the second used computed tomography and had a three week follow up.

\section{Epoprostenol (prostacyclin) and its analogues}

Intravenous infusion of prostacyclin causes systemic vasodilatation (especially in the skin) and inhibits platelet aggregation. After several small studies, a randomised controlled trial of 86 patients with ischaemic strokes confirmed by computed tomography showed no benefit. ${ }^{5}$ Stable analogues of prostacyclin are currently under evaluation.

\section{Full dose anticoagulants}

Full therapeutic doses of heparin and oral anticoagulants have been used to treat acute stroke for many years, with continuing controversy about the balance of benefits (prevention of thromboembolic extension or recurrence) and risks (bleeding, even when computed tomography is used to exclude non-ischaemic stroke). Recent controlled studies have not shown overall benefit in patients with or without potential cardiac sources of embolism. ${ }^{2}$ Pending results of larger trials, the use of full dose anticoagulants is not recommended. The defibrinating enzyme, ancrod, reduces plasma fibrinogen concentrations and thus reduces plasma and blood viscosity as well as producing anticoagulation, but only one small study has been reported.

\section{Low dose heparin and heparinoids}

Deep venous thrombosis is common in patients with acute hemiparetic stroke, and pulmonary thromboembolism is an important cause of death. Dextran 40 was ineffective in preventing deep vein thrombosis in a subgroup of patients in the Scandinavian haemodilution study. ${ }^{2}$ Recent studies have shown that low dose subcutaneous standard heparin or the heparinoid ORG 10172 are effective in reducing the incidence of deep vein thrombosis in patients with acute stroke and do not cause appreciable bleeding. Though one large study that did not use routine computed tomography also reported reductions in pulmonary embolism and in total deaths,${ }^{6}$ computed tomography to exclude intracranial haemorrhage is advisable before using even low dose anticoagulants Further large trials of low dose heparin, heparin fractions, and heparinoids are certainly required. 
Thrombolytics

Treatment with thrombolytics risks causing cerebral bleeding and cerebral oedema, and its use is still experimental.

\section{INTRACRANIAL HAEMORRHAGE}

No medical treatment has been proved to be beneficial against intracerebral haemorrhage; a study of dexamethasone showed no benefit. ${ }^{2}$ Two studies of subarachnoid haemorrhage showed that the fibrinolytic inhibitor, tranexamic acid, reduced the incidence of rebleeding but increased the incidence of delaved cerebral ischaemia and did not reduce death rates. ${ }^{\circ}$ Controlled trials of nimodipine, however, showed significant reduction in neurological deficits after cerebral vasospasm. ${ }^{8}$ Nicardipine is also under evaluation. It may be logical to combine calcium entry blockade with fibrinolytic inhibition in future studies.

\section{PRIMARY PREVENTION OF STROKE}

A recent community based study of first ever stroke caused by cerebral infarction confirmed that hypertension preceded over half the strokes. The most efficient drug treatment to prevent stroke is probably to give older hypertensive patients (with diastolic blood pressure persistently over $100 \mathrm{~mm} \mathrm{Hg}$ ) a thiazide diuretic. ${ }^{9}$ According to the trial of the European working party on high blood pressure in the elderly, treating 100 older hypertensive patients for two years can prevent two fatal and two non-fatal cardiovascular events, especially stroke. Treating younger patients with similar blood pressure levels is less efficient, though the MRC working party on treating mild hypertension suggested guidelines for treating younger hypertensive people who may benefit more-for example, with bendrofluazide for men aged 55-64 who smoke or with propranolol for non-smoking men. ${ }^{10}$

The lack of suitable screening tests to indicate increased platelet reactivity, which would be analogous to measuring blood pressure, hinders the use of aspirin or other antiplatelet agents. Despite this disadvantage, two studies of aspirin treatment as primary prevention of cardiovascular events showed that fewer treated people experienced cardiovascular events, including strokes." On the other hand, long term aspirin treatment possibly increases the incidence of haemorrhagic or disabling stroke, as well as having the proved side effect of gastrointestinal bleeding."

Recent evidence suggests that oestrogen replacement therapy may reduce the incidence of stroke in postmenopausal women.

\section{SECONDARY PREVENTION OF STROKE}

About one third of patients admitted to hospital die soon after their stroke, and another third have major disability. The remaining third make a complete or good recovery, however, and it is important to establish whether drugs can reduce the risk of subsequent cardiovascular events. Although only about $15 \%$ of strokes are preceded by a transient ischaemic attack, such events are worrying for patients and doctors, and the value of drugs to prevent the completion of a stroke should also be considered. An overview of trials of antiplatelet treatment in patients with transient ischaemic attacks or minor ischaemic strokes (as in patients with unstable angina or myocardial infarction) showed that $15 \%$ fewer treated patients died and $30 \%$ fewer had non-fatal strokes or myocardial infarctions. ${ }^{13}$

Aspirin ( $300 \mathrm{mg}$ a day) appears to be as effective as any other antiplatelet treatment. Lower doses of aspirin (50-150 mg a day) might in theory have a higher ratio of benefit to risk, but there is no evidence vet that lower doses prevent secondary strokes. It is therefore inappropriate for the current British National Formulary to suggest a dose of $75 \mathrm{mg}$ a day. Lower doses might reasonably be tried, however, for patients with gastric intolerance of the standard $300 \mathrm{mg}$ dose that is unrelieved by using an enteric coated preparation. Doctors who do not (or cannot) institute computed tomography for patients with stroke to exclude the $10-15 \%$ with intracranial haemorrhage should appreciate that aspirin may cause further bleeding in these patients. ${ }^{14}$

As for other antiplatelet drugs, little evidence currently exists that sulphinpyrazone or dipyridamole are effective in preventing secondary stroke when used without aspirin, but dipyridamole is being compared with low dose aspirin in a current large study. Ticlopidine, a newer antiplatelet agent, has been assessed in two trials of prevention after a stroke or transient ischaemic attack (CATS and TASS), whose results will be published shortly. The high percentage of patients who cannot tolerate aspirin makes it necessary to evaluate other antiplatelet agents.

Another review suggested that treating hypertension in patients with transient ischaemic attacks or minor strokes may also reduce subsequent strokes by up to half. ${ }^{\times}$Treatment with antihypertensives as well as aspirin should therefore be considered.

\section{VASCULAR (MULTI-INFARCT) DEMENTIA}

This continues to be a controversial subject. Vascular dementia is less common than Alzheimer's (senile) dementia, their differential diagnosis can be difficult, and assessing drugs to treat them is difficult. There is no convincing evidence that vascular drugs make an important contribution to the care of demented patients.

\section{Peripheral arterial disease}

INTERMITTENT CLAUDICATION

In this common condition of older people, the prognosis for the legs is relatively benign and fewer than $10 \%$ progress to critical ischaemia (rest pain or gangrene). The general prognosis gives cause for concern, however, because the associated coronary and cerebral arterial disease carry a high risk of fatal or non-fatal myocardial infarction or stroke. Intervention to prevent vascular events and death should therefore be as important as treating the cramping pain. Yet studies of preventing secondary cardiovascular disease in patients with claudication are surprisingly rare. No large studies have been undertaken to assess the clinical benefit of lowering either blood pressure or serum lipid concentrations in patients with claudication. When treating hypertension it may be preferable to avoid $\beta$ adrenergic blocking drugs because some studies showed them to have adverse effects on walking distance and circulation in the legs. No large studies of the clinical benefit of aspirin in patients with claudication have been undertaken, although a reduction in the progression of femoral atherosclerosis was observed in one study of low dose aspirin. A recent study of oral anticoagulants in recipients of limb grafts showed significantly improved survival of the patients (if not the grafts), which, if this result is confirmed, may lead to a renaissance in anticoagulant treatment for patients with claudication.

The recently reported PACK (prevention of atherosclerotic complications with ketanserin) study was a large placebo controlled trial of this serotonin antagonist to prevent cardiovascular events in patients with intermittent claudication. ${ }^{15}$ Ketanserin has antihypertensive, antiplatelet, and rheological actions. Patients using potassium losing diuretics, however, experienced drug interactions associated with a higher death rate, which counteracted a beneficial trend in the 
other patients. This study has, however, provided a useful basis for planning trials of other interventions in patients with claudication, which might reduce their risk of vascular events. Of two smaller studies of ticlopidine in preventing vascular events in patients with claudication, one has been reported ${ }^{16}$ and the second (STIMS) is expected to be reported shortly.

The placebo group in the large PACK study underwent serial treadmill walking distance studies in several centres, and the results confirm the findings of previous studies that walking distance tends to improve spontaneously, noticeably, and for several months, especially if patients are encouraged to "stop smoking and keep walking." Evaluating drugs in relieving the symptoms of claudication is therefore difficult: strict selection and large numbers of patients are required. Despite the statement in the current British National Formulary that "no controlled studies have shown any improvement in walking distance," large controlled studies have shown positive results for isoxsuprine, naftidrofuryl, ${ }^{17}$ oxpentifylline, ${ }^{1 .}$ and ticlopidine. ${ }^{\text {to }}$ Though the mean increase in walking distance compared with that in patients receiving placebo is often small, some individual patients do show impressive responses. One or more of these agents may therefore be worth trying in patients whose daily activities are severely curtailed, while the anticipated benefits of time, regular exercise, and stopping smoking are awaited. Though little evidence exists that vasodilatation in itself increases blood flow to ischaemic leg muscles, several other mechanisms (such as rheological or metabolic actions) have a sound rationale. Furthermore it seems inappropriate to have a double standard for claudication and angina pectoris, whose placebo response is also well documented. Patients are as frustrated if they cannot walk because of a sore lower half as because of a sore upper half, and doctors who discriminate at the umbilicus may hit below the belt.

The theoretical importance of blood viscosity in intermittent claudication has been substantiated by a recent controlled trial of haemodilution in selected patients with claudication who have haemodynamically appropriate lesions (long stenoses) that might be expected to result in low shear stresses, under which the increased viscosity of blood may become a limiting factor in muscle blood flow. Haemodilution is not "user friendly" but should be considered in severely disabled patients with claudication who do not respond to simpler measures and have no contraindications (such as severe ischaemic heart disease).

The most obvious way to relieve claudication is to relieve haemodynamically important obstructions in major arteries. Bypass grafting carries a risk of death of $0 \cdot 5-3 \%$, and grafts fail progressively in time, especially in smokers. Recent alternatives in selected cases include angioplasty (balloon or laser) and treatment with thrombolytic drugs. Recent acute onset or worsening of claudication suggests thrombosis of a stenosed artery, and angiography may give evidence of thrombosis that has been confirmed in some cases by reperfusion after thrombolytic treatment. The current popularity of thrombolytics to treat acute myocardial infarction has promoted a renaissance of interest in applying thrombolytic treatment to subacute as well as acute limb ischaemia. ${ }^{14}$ The main adverse effect is bleeding, of which the most serious manifestation is the death of about $1 \%$ from intracranial haemorrhage (for example, from an unsuspected intracranial aneurysm). This death rate, however, is comparable with that risked by operating and may be reduced by modifications currently being investigated. The modifications include local infusion of the thrombolytic agent proximal to or into the thrombus via the catheter used to show the lesion by angiography and combining thrombolytic treatment with aspirating thrombus fragments to shorten the duration of treatment.

\section{CRITICAL ISCHAEMIA}

This may arise acutely (because of major thromboembolism) or subacutely (caused by progressive occlusion at a critical stenosis, for example, by overlying thrombus formation). Though embolectomy, angioplasty, and bypass grafting are used as appropriate, there is also increasing interest in treatment with thrombolytics, including local infusions proximal to or into the thrombus. ${ }^{14}$

Some evidence also exists that infusions of vasodilators such as inositol nicotinate, naftidrofuryl, and prostanoids (prostaglandin $\mathrm{E}_{1}$, epoprostenol (prostacyclin, prostaglandin $\mathrm{I}_{2}$ ), or stable prostacyclin analogues) may relieve subacute rest pain or promote ulcer healing, or both ${ }^{20}$; however, the results of large controlled studies are awaited before treatment with vasodilators can be recommended with confidence. As with intermittent claudication, several mechanisms exist by which vasodilatation may act (table I), which can be helpful in "buying time" while patients are evaluated for surgery, angioplasty, or thrombolytic treatment. As with claudication, the main problem in trials is the response of rest pain and ulceration to placebo treatment and the passage of time.

\section{RAYNAUD'S DISEASE}

The mainstays of treatment of Raynaud's disease are general measures such as keeping warm, wearing gloves (including heated gloves), and excluding causes such as drugs, vibrating tools, and diseases of the cervical ribs or connective tissue. Drug treatment should be considered for severe cases, and placebo controlled trials of nifedipine, inositol nicotinate, and prostanoids suggest that they are of benefit." The benefit of these vasodilators is, however, often tempered by their systemic side effects (table I). ${ }^{21}$

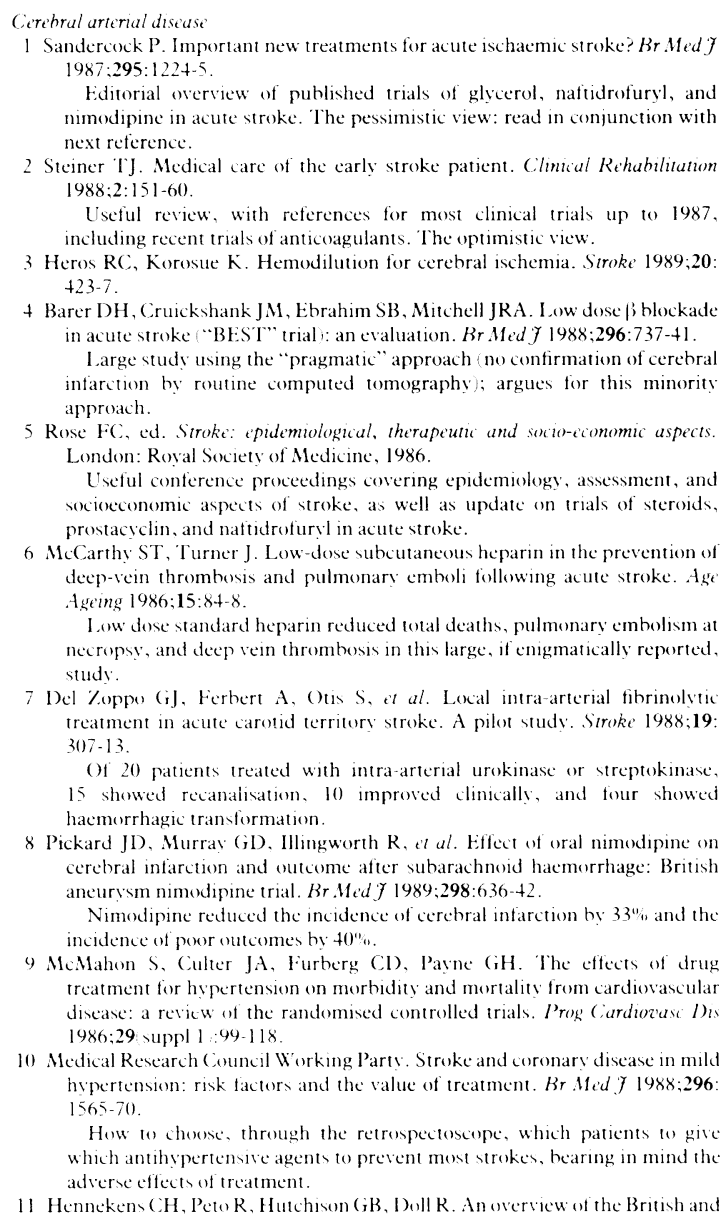

6 McCarthy ST, Turner J. Low-dose subcutaneous heparin in the prevention of deep-vein thrombosis and pulmonare emboli following acute stroke. A te Aseing 1986;15:84-8.

Low dose standard heparin reduced total deaths, pulmonary embolism at necronsy, and deen vein thrombersis in this large, if enigmatically reported. study

7 Del \%oppo (i). Ferhert A, Otis S, et al. Lecal intra-arterial fibrinolvtic traament in acute carotid territory stroke. A pilot studv. Stroke 1988;19: $307-13$.

Of 20 patients treated with intrararterial urokinase or streptokinase. 15 showed recandication, 10 improved dinicalls, and four showed hacmorrhagic transformation.

8 Pickard JD, Murray (GD, Illingworth R, et al. Effect of oral nimodipine on cerebral infarction and outcome after subarachnoid hacmorrhage: British aneurysm nimodipine trial. Br. Med J 1989;298:636-42

Nimodipine reduced the incidence of cerebral infarction by $33 \%$ and the incidence of poor outcomes by $40 \%$.

9 McMahon S, Culter JA, Furberg CD), Payne (iH. The effects of drug treatment for hypertension on morbidity and mortalits from cardiovascular discase: a revicw of the randomised controlled trials. I'rog (Cardioves I) 1986:29 suppl $1: 99-118$

10 Medical Research Council Working Parts: Stroke and coronary disease in mild hypertension: risk factors and the value of treament. Br Med 7 1988;296: hyperter $1565-70$

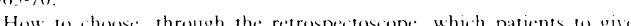
How to choose, through the retrospectoscope, which patients to give which anthypertenstec agents to pretent most strokes, hearing in mind the 11 Hennekens (CH, Peto R, Hutchison (iB, Dell R. Anoversiew of the British and 
American aspirin studies. $N$ Engl F Med 1988;318:923-4

Overview of the two large primary prevention studies of aspirin

12 Paganini-Hill A, Ross RK, Henderson BE. Postmenopausal oestrogen

treatment and stroke: a prospective study. Br Med f 1988;297:519-22.

An interesting spin off of oestrogen replacement treatment: deaths from stroke roughly halved.

13 Antiplatelet Trialists' Collaboration. Secondary prevention of vascular disease by prolonged antiplatelet treatment. Br Med f 1988;296:320-1.

14 Sandercock P. Aspirin for strokes and transient ischaemic attacks. No panacea. Br Med f 1988;297:995-6.

Counsels appropriate caution before reflex prescription of aspirin for all strokes.

Peripheral arterial disease

15 Prevention of Atherosclerotic Complications with Ketanserin Trial Group. Prevention of atherosclerotic complications: controlled trial of ketanserin. BrMed f 1989;298:424-30

Largest study of patients with claudication (3899 patients) and their natural history.

6 Arcan JC, Blanchard J, Boissel JP, Destors JM, Panak E. Multicenter doubleblind study of ticlopidine in the treatment of intermittent claudication and the prevention of its complications. Angiology 1988;39:802-11.

Ticlopidine for six months increased walking distance and reduced cardiovascular events in this small study ( 169 patients).

17 Trubestein $\mathrm{G}$, Böhme $\mathrm{H}$, Heidrich $\mathrm{H}$, et al. Naftidrofuryl in chronic arterial disease. Results of a controlled multicentre study. Angiology 1984:35:701-8.
In 104 patients treated for 12 weeks placebo and active treatment gave similar increases in total walking distance, but active treatment was associated with a greater increase in pain free walking distance.

18 Rössner M, Müller R. On the assessment of the efficacy of pentoxifylline (Trental). F Med 1987;18:1-15.

In house meta-analysis of the efficacy of oxpentifylline in increasin walking distance in claudication in 14 double blind randomised studies. In total, $35 \%$ of patients receiving active treatment doubled their walking dist, 3 \% of pation $8 \%$ active treatment

19 Graor RA, Risius B, Denny KM et al. Local thombolysis in the treatment of thrombosed arteries, bypass grafts and arteriovenous fistulas. $\mathcal{F}$ Vasc Surg 1985;2:406-14.

Large series of patients treated with low dose intra-arterial streptokinase; discusses efficacy, coagulation changes, complications, and choice of patients

20 Negus D, Irving JD, Fiedgood A. Intra-arterial prostacyclin compared to Praxilene in the management of severe lower limb ischaemia: a double-blind trial. $\mathcal{F}$ Cardiovasc Surg (Torino) 1987;28:196-9.

After either treatment about half the patients had symptomatic relief and avoided amputation. Discusses previous studies of both epoprostenol and naftidrofuryl in critical ischaemia.

21 Corbin DOC, Wood DA, Macintyre CCA, Housley E. A randomised double-blind cross-over trial of nifedipine in the treatment of primary Raynaud's phenomenon. Eur Heart f 1986; 7:165-70.

Treatment reduced number of attacks in most patients; $61 \%$ had side effects (especially flushing and acroparaesthesiae).

\section{Scientifically Speaking}

\section{A real washday hazard}

\section{Bernard Dixon}

Some years ago that most elegant physicist, historian of science, and war time boffin par excellence, RV Jones wrote a memorable account of what he termed self frustration. Thus the Germans, before mounting their V2 attacks on London in 1944, took considerable trouble to deceive the Brits about the number of rockets stored in forward areas in France. They did so in part by constructing dummy sites, ready to be photographed by enemy reconnaissance planes. Whereas the real sites were difficult to find, all of these were easily and quickly spotted-as intended. Then the Brits captured a map revealing the locations of genuine sites. But joy was short lived because these were all west of the river Seine and the allies knew that most of the actual sites were east of the river. Intelligence was applied. Perhaps the Germans had deployed dummies and genuine sites in consistent proportions. The assumption was made, it proved correct, and London soon learnt the size of the firepower about to be unleashed. This would not have been possible had the Germans not set up their machinery of deception.

Likewise the story of Lord Cherwell, personal scientific adviser to Winston Churchill. As the super boffin, Cherwell had a special pass, intended to give him immediate access to all government establishments during the war. Unlike the passes carried by more numerous officials of lower rank, this type was limited to a tiny handful of exceedingly important people. Moreover, its appearance was kept confidential so that it could not be forged. As a result Lord Cherwell had frequent and furious arguments with guards at top secret buildings around the country. Never having seen such a pass, they refused to let its impatient and self important holder enter their premises.

\section{Welter of self frustration}

Now let me venture a prediction-that our contemporary preoccupation with green, ecologically sound policies is about to throw up a welter of self frustration. Whether on the grand scale of global warming and the hole in the ozone or in more manageable matters of garden pests and organic eating, it seems highly likely that we shall be continually taunted by the unintended and unanticipated consequences of actions which in themselves are both wholesome and desirable.

My prediction is confident. But it is not brave, because confirmatory evidence is already coming to light. Consider, for example, laundering and the propensity of bacteria to stick to the cloth of trousers and socks, pyjamas and underwear, tablecloths and teatowels. Researchers in the Institute of Medical Microbiology at the University of Milan have been pursuing this issue recently. Their objective - to determine whether fabric washing achieves such immaculate results microscopically as it often does macroscopically, or whether it is a potentially significant route for the dissemination of micro-organisms. In the case of hospitals, schools, hotels, and the like, this is a question well worth asking.

Carlotta Granucci and her colleagues have now reviewed the literature and presented their own experimental findings. The picture is not encouraging. Many different types of micro-organism can stick avidly to fabrics. They include viruses, pathogenic bacteria, fungal spores, and protozoan trophozoites and cysts. Some find sufficient moisture and nutrients to grow and form colonies capable of rotting the material.

Others are washed away. And in grandmother's day the piping hot temperatures used for the weekly wash and in commercial laundries were sufficient to sterilise the garments of all but the most hardy spores. But now comes the rub, in the form of two ecological concerns. Firstly, growing anxiety about energy saving has triggered a shift away from washing with scalding fingers towards much lower temperatures, which are tolerated by most micro-organisms. Secondly, biologists have traced the deadening of rivers and lakes by eutrophication to substances in waste water discharged into these natural water systems. A key culprit has proved to be sodium tripolyphosphate, which in consequence has been removed from commercial laundry detergents, or at least drastically reduced in concentration. But the main role of sodium tripolyphosphate in laundering was to prevent organic and inorganic substances from accumulating on fabrics. Washed repeatedly without it, shirts, socks,
Bernard Dixon, PHD, science writer 